\title{
Matemática financeira e planilhas eletrônicas: uma abordagem com a incorporação de recursos computacionais
}

\author{
Financial mathematics and electronic spreadsheets: an approach to the incorpora-tion \\ of computational resources
}

\author{
Rodrigo Duda ${ }^{* 1}$ e Luciane Grossi ${ }^{2}$ \\ ${ }^{1}$ Instituto Federal do Paraná - Câmpus Irati, PR - Brasil \\ ${ }^{2}$ Universidade Estadual de Ponta Grossa, PR - Brasil
}

\begin{abstract}
Resumo
Este trabalho tem por objetivo propor uma abordagem alternativa para o ensino de progressões e matemática financeira, com a incorporação de recursos tecnológicos. Um dos conceitos utilizados é o estudo de progressões aritméticas e geométricas. Para o estudo destes, propomos a abordagem por meio da resolução de relações de recorrência. Com o objetivo de estimular a manipulação algébrica e a compreensão das variáveis das fórmulas de matemática financeira, propomos a incorporação de recursos tecnológicos, em particular, das planilhas eletrônicas, nas aulas sobre o tema. Por meio dessa ferramenta, propomos a viabilização da construção de simuladores de matemática financeira em sala de aula, visando a diversificação das atividades.
\end{abstract}

Palavras-chave: Matemática financeira. Progressões e relações de recorrência. Planilhas eletrônicas.

\begin{abstract}
This paper has as its goal to propose a new alternative approach to the progressions and financial math teaching, with the incorporation of technological resources. One of the concepts that used is the study of geometric and arithmetic progressions. In order to study those, an approach based on recurrence relations is proposed. With the goal of stimulating the algebraic manipulation and understanding of the financial math formulas variables, it is proposed the incorporation of the technological resources, in particular, of electronic spreadsheets, in classes about the theme. Through the tool, it is proposed the viability of financial math simulators in the classroom, seeking the diversification of activities.
\end{abstract}

Keywords: Financial math. Progressions and recurrence relations. Electronic spreadsheets. 


\section{Introdução}

Uma alternativa viável para diversificação das estratégias docentes é a utilização de ferramentas tecnológicas em sala de aula, em especial, o uso de computadores.

Muitos são os pesquisadores favoráveis ao seu uso no ambiente escolar, pois além de ser uma ferramenta que se torna cada vez mais frequente na vida dos educandos, sua utilização pode englobar tanto a pesquisa acerca da temática estudada quanto a utilização de softwares educacionais ou de execução de cálculos, constituindo-se num grande leque de possibilidades à disposição do professor.

A presente proposta se baseia na revisão de literatura sobre as temáticas Ensino de Matemática Financeira e Recursos Computacionais no Ensino de Matemática, buscando relacionar a matemática escolar com o cotidiano, bem como a diversificação de atividades por meio da utilização de ferramentas tecnológicas.

Neste contexto, destacamos a abordagem do estudo de progressões aritméticas e geométricas por meio da utilização de relações de recorrência de primeiro grau, a aplicação de progressões e relações de recorrência na modelagem e resolução de situaçõesproblema relacionadas à matemática financeira e a utilização de planilhas eletrônicas na construção de calculadoras e simuladores para aplicação em alguns tópicos de matemática financeira.
As diretrizes curriculares, tanto nacionais quanto estaduais, colocam a educação escolar como agente transformador do aluno, seja ela no tocante a sua vida acadêmica, à interpretação do mundo que o rodeia, ao mundo do trabalho, ou ainda, ao exercício da cidadania.

Desta forma, buscamos uma alternativa para que a aprendizagem deste importante tema da matemática seja efetivamente mais significativa, de forma que seu estudo não seja pautado apenas na abordagem dos sistemas de capitalização simples e composta, tampouco pautado na resolução mecânica de exercícios referentes à temática.

\section{Referencial teórico}

A seguir apresentamos a revisão bibliográfica e o referencial teórico utilizado na estruturação do presente trabalho, no tocante ao papel da matemática na formação do educando, à importância da matemática financeira para o cidadão e à possibilidade de incorporação de recursos tecnológicos nas aulas de matemática.

\subsection{O ensino de matemática financeira e sua função social}

Entre as finalidades da Educação Básica descritas no Art. $22^{\circ}$ da Lei no 9394/96 (LDB), que estabelece as diretrizes e bases da Educação Nacional, figura o desenvolvimento do educando, assegurando-lhe formação indispensável para o exercício da cidadania. Nesse sentido, cabe destacar 
que o bom entendimento sobre as práticas comerciais faz parte do que engloba o conceito de cidadania.

Ser cidadão implica estar sujeito a uma série de deveres e direitos. Dentre os últimos, a qualidade de vida do indivíduo está amplamente relacionada, pois o bom uso do dinheiro certamente acarretará uma melhoria de condições, principalmente no que diz respeito ao não endividamento e ao consumo consciente, bem como verificação se os seus direitos fundamentais enquanto consumidor estão sendo respeitados.

A preocupação sobre o assunto figura também entre as recentes iniciativas políticas. O Governo Federal, por meio do Decreto № 7.397, de 22 de dezembro de 2010, instituiu a Estratégia Nacional de Educação Financeira (E$\mathrm{NEF}$ ), visando a promoção da educação financeira e previdenciária para o fortalecimento da cidadania, a eficiência e solidez do sistema financeiro nacional e a tomada de decisões conscientes por parte dos consumidores.

Neste sentido, é fundamental que esse tema também seja abordado no ambiente escolar. No atual estágio econômico do Brasil, no qual uma grande parcela da população tem acesso facilitado a diferentes modalidades de crédito, cabe ao ensino básico de matemática oferecer aos alunos uma formação sólida referente à temática financeira (GIRALDO, V; CAETANO, P; MATTOS, F., 2012, p. 45).

Segundo as Diretrizes Curriculares para a Educação Básica do Estado do Paraná para a disciplina de Matemática (2008, p.61), é importante que o aluno do Ensino Médio compreenda a matemática financeira em diferentes contex- tos da atividade humana, como o trato com dívidas, descontos em crediários, interpretação de descontos, compreensão de reajustes salariais e escolha de aplicações financeiras.

Desta forma, torna-se óbvio que a necessidade de conhecimento matemático sobre a temática é essencial para guiá-lo na tomada de decisões, que certamente contribuirão para uma melhor compreensão sobre o uso do dinheiro.

Essa visão também é apontada por Giraldo; Caetano e Mattos (2012, p. 45). Os autores afirmam que a Matemática Financeira aplicada aos diferentes ramos da atividade econômica representam um importante instrumento auxiliar em decisões de ordem pessoal e social.

Para Santos, R. (2011, p. 19), os conteúdos e estratégias de Matemática Financeira são fundamentais para um ensino em que se estimule a investigação e criticidade do cidadão, em detrimento à descrição de algoritmos, fórmulas e cálculos descontextualizados.

Segundo SOUSA (2012, p.21), "[...] uma população educada financeiramente pode modificar a realidade financeira de um lugar." Para isso, o autor aponta que é necessário, além de gerir os recursos de forma otimizada, compreender as relações econômicas, que nem sempre estão visíveis ao cidadão.

Enaltecendo essa visão acerca da interpretação de financiamentos, Morgado; Wagner e Zani (2001, p. 44) apontam que a operação básica da matemática financeira é a operação de empréstimo. Desta forma, é de se esperar que o enfoque do ensino de matemática financeira no Ensino Médio englobe situações que contemplem tais operações, e não 
somente o cálculo básico de juros e porcentagens.

Em resumo, as características e conclusões anteriores conduzem a um caminho onde se torna necessário o uso de ferramentas matemáticas para melhorar a compreensão sobre conceitos básicos de matemática financeira.

Nessa perspectiva, vemos a importância da compreensão dos conceitos básicos envolvidos em operações financeiras, em especial, no universo dos empréstimos e financiamentos disponíveis ao consumidor, visando o uso consciente do dinheiro e a aplicação do conhecimento para a tomada de decisões.

\subsection{Recursos computacionais e ensi- no de matemática}

Segundo Penteado (2000, p. 23), "Para explorar o potencial educacional das Tecnologias Informáticas (TI), é preciso haver mudanças na organização da escola e, particularmente, no trabalho do professor." Neste sentido, a resistência do docente à introdução de novas abordagens em sala de aula, bem como sua preparação técnica para tal desafio são aspectos que interferem qualitativamente em atividades desse cunho. Tais mudanças "[...] afetam a zona de conforto da prática do professor e criam uma zona de risco caracterizada por baixo índice de certeza e controle da situação de ensino" (PENTEADO, 2000, p. 23).

Segundo Gravina e Basso (2012, p.12-13), o desenvolvimento da sociedade e de tecnologias são processos que se realimentam, sendo que temos na tecnologia digital uma ampliação de possibilidades para "experimentos de pensamento", quando comparadas aos textos e desenho estático, disponibilizando ferramentas que suportam a exteriorização, diversificação e ampliação de pensamentos.

Borba e Penteado (2005, pg. 48), apontam que a informática "permite que a linearidade de raciocínios seja desafiada por modos de pensar". Esse aspecto baseia-se na simulação e experimentação, envolvendo escrita, oralidade, imagens e comunicação instantânea, dentro de uma nova linguagem.

Nessa perspectiva, cabe salientar que deve ser dada liberdade para que os alunos possam fazer a análise dos objetos estudados e é necessário o estímulo para a investigação, pois o aspecto de possibilidades de simulação se torna um diferencial da abordagem por meio do uso de recursos computacionais.

O fato de poder simular situações pode favorecer a percepção de aspectos que normalmente não são percebidos pelo aluno durante a resolução de situações-problema, além de possibilitar explorações que por limitações temporais poderiam não ser abordadas em sala.

\subsection{Matemática financeira e progres- sões}

Conforme citado anteriormente, entre as finalidades da educação básica estão incluídos o pleno desenvolvimento do educando e o seu preparo para o exercício da cidadania. Desta forma, espera-se que os conteúdos estejam inter-relacionados, de forma que o estudo desses não seja meramente pautado na resolução de situações-problema sem 
sentido prático, mas que estas sejam contextualizadas.

Lima (2007, p. 171), destaca que "São praticamente inesgotáveis as possibilidades de enriquecer os livros didáticos". O autor aponta, entretanto, que muitos exercícios, em diferentes temáticas, são limitados a práticas manipulativas, problemas artificiais e até mesmo aplicações incabíveis atualmente.

No que diz respeito ao ensino de Progressão Aritmética (PA) e Progressão Geométrica (PG), por exemplo, Lima et. al. (2006, p. 40-43), apontam como desnecessários os seguintes tópicos:

a) Relação entre três termos consecutivos de uma PA ou PG;

b) Fórmulas para facilitar o cálculo da razão, número de termos e $1^{\mathrm{o}}$ termo de uma progressão;

c) Cálculo do produto dos termos de uma PG;

d) Problemas envolvendo soma ou diferenças de dois termos quaisquer de uma PA ou PG.

Os autores também destacam a necessidade do uso da calculadora para a resolução de problemas reais envolvendo progressões geométricas e a importância da relação entre progressões geométricas e a ideia de taxa de crescimento constante.

A compreensão sobre a ideia de taxa de crescimento constante é primordial para o entendimento das noções iniciais sobre o cálculo de juros compostos. Embora esse conceito possa ser explorado em diferentes contextos, a temática monetária talvez seja a que mais chame a atenção do aluno do Ensino Médio, pois estes já possuem anseios referentes ao mundo do trabalho, sendo a situação financeira familiar e as incli- nações pessoais fatores influentes no momento de decidir entre a carreira $\mathbf{u}$ niversitária e o ingresso no mercado de trabalho (LIMA, 2007, p. 168).

\subsection{Progressões e relações de recorrên- cia}

Conforme as Orientações Educacionais Complementares aos Parâmetros Curriculares Nacionais para as Ciências da Natureza, Matemática e suas Tecnologias (2000, p. 122), na área de matemática, entre as unidades temáticas do eixo estruturante "Álgebra: números e funções" figura a temática "sequências numéricas: progressões e noção de infinito".

Dentro desse tema, em livros didáticos normalmente são apresentadas somente as progressões aritméticas e as geométricas. No entanto, há a abertura para uma abordagem diferenciada do estudo de progressões, que pode ser realizada por meio da resolução de relações de recorrência.

Tal abordagem se torna necessária para que os alunos possam modelar situações-problema utilizando equações de diferenças de primeira ordem, as quais não apresentam maior complexidade se comparadas às PAs e PGs e, em alguns casos, constituem-se na maneira mais prática para a modelagem de determinados problemas.

2.4.1 Relações de recorrência de primeira ordem

Algumas sequências podem ser definidas recursivamente, ou seja, por meio de uma regra que permite expressar qualquer termo da sequência em função de seus antecessores. Essas re- 
gras, ou relações, são chamadas de relações de recorrência ou, simplesmente, recorrências.

Uma recorrência é dita de primeira ordem quando o termo $x_{n+1}$ é expresso em função do termo $x_{n}$. Tal recorrência será linear se, e somente se, a função de $x_{n}$ for do primeiro grau (LIMA et al., 2006).

Desta forma, são exemplos de recorrências lineares:

$$
\begin{gathered}
x_{n+1}=2 x_{n}, \\
x_{n+1}=-3 x_{n}+4, \mathrm{e} \\
x_{n+1}=x_{n}-1 .
\end{gathered}
$$

\subsection{Progressões aritméticas e relações de recorrência}

Sugerimos a introdução da temática de sequências pela notação de relações de recorrência como elemento desafiador para que os alunos possam escrever a expressão que exprime uma progressão aritmética apenas pela sua definição, em detrimento à apresentação direta deste tópico pelo professor.

Para isso, será necessário o conhecimento acerca da resolução de relações de recorrência, ou seja, obter uma função de $n$ com a qual seja possível calcular o valor de qualquer $x_{n}$ sem utilizar a recorrência original.

A seguir, apresentamos como esse conhecimento pode se utilizado no estudo de progressões aritméticas.

Definição 1: Uma progressão aritmética é uma sequência numérica na qual a diferença entre dois termos consecutivos quaisquer é sempre igual a uma constante $r$, a qual chamamos de razão da progressão (MORGADO; WAGNER, ZANI, 2001, p. 01).
Pela Definição 1, podemos reescrever os termos de uma PA, de primeiro termo igual a $a_{n}$ e razão igual a $r$ como:

$$
\begin{gathered}
a_{2}=a_{1}+r \\
a_{3}=a_{2}+r \\
\vdots \\
a_{n-1}=a_{n-2}+r \\
a_{n}=a_{n-1}+r
\end{gathered}
$$

Somando os membros das igualdades ordenadamente, e subtraindo o termo $a_{2}+a_{3}+\ldots+a_{n-1}$ em ambos os membros, obteremos (1):

$$
a_{n}=a_{1}+(n-1) \cdot r
$$

Desta forma, (1) é a resolução da relação de recorrência relacionada à progressões aritméticas, permitindo o cálculo de qualquer valor de $a_{n}$ em função de $n$.

A abordagem de progressões por meio de relações de recorrência pode ser facilitadora do entendimento de outras relações entre os termos da progressão. O raciocínio utilizado para calcular a expressão que determina o termo geral de uma PA pode ser utilizado não necessariamente escrevendo a PA iniciando por $a_{1}$.

Partindo da escrita da progressão a partir do termo $a_{k-1}$, pode ser explorada a relação $a_{n}=a_{k-1}+(n-k+1) r$, por meio da qual é possível relacionar quaisquer termos de uma progressão aritmética.

2.5.1 Soma dos termos de uma PA finita

Um fato histórico bastante peculiar acerca de progressões aritméticas é a fórmula que expressa a soma dos termos de uma PA. Carl Friedrich Gauss, 
aos sete anos de idade, surpreendeu seu professor ao apresentar a soma dos números naturais de 1 a 100 em poucos minutos. Seu professor, esperando que o trabalho levasse pelo menos uma hora, surpreendeu-se quando o jovem Gauss apresentou a soma, que tem como resultado o número 5050 .

Gauss explicou que para tal feito efetuou as somas 1+100, 2+99, 3+98, e assim por diante, de forma que obteve 50 respostas iguais a 101. Desta forma, o resultado da soma é igual a 50 vezes 21 , ou seja, 5050 (MORGADO; WAGNER, ZANI, 2001, p. 04).

O raciocínio utilizado por Gauss consiste em uma manipulação envolvendo a escrita da soma dos $n$ primeiros termos da PA em ordem posicional decrescente. Sendo $S_{n}$ a soma procurada, podemos escrevê-la de duas formas, conforme indicado em (2) e (3):

$$
\begin{aligned}
& S_{n}=a_{1}+a_{2}+\ldots+a_{n-1}+a_{n} \\
& S_{n}=a_{n}+a_{n-1}+\ldots+a_{2}+a_{1}
\end{aligned}
$$

Somando ordenadamente os termos de (2) e (3), obteremos (4):

$$
2 S_{n}=\left(a_{1}+a_{n}\right)+\ldots+\left(a_{1}+a_{n}\right)
$$

Os demais pares de somas em (4) são do tipo $a_{p}+a_{n-p+l}$. Usando (1), essas somas podem ser reescritas conforme indicado em (5):

$$
a_{1}+(p-1) r+a_{1}+(n-p+1-1) r
$$

Efetuando as simplificações em (5), obtemos (6):

$$
a_{1}+a_{1}+(n-1) r
$$

Desta forma, por (1), obtemos que todos os pares de somas de (4) são constantes e iguais a $a_{1}+a_{n}$, aparecendo $n$ vezes no segundo membro da igualdade. Com isso, obtemos (7):

$$
2 S_{n}=\left(a_{1}+a_{n}\right) n
$$

Desta forma, a soma dos $n$ primeiros termos de uma PA é dada por (8):

$$
S_{n}=\frac{\left(a_{1}+a_{n}\right) n}{2}
$$

\subsection{Progressões geométricas e relações de recorrência}

Assim como para a determinação da expressão que exprime uma PA, as recorrências podem ser utilizadas para a determinação da expressão que exprime os termos de uma PG.

Definição 2: Uma progressão geométrica é uma sequência numérica na qual o quociente entre dois termos consecutivos quaisquer é sempre igual a uma constante real $q$, a qual chamamos de razão da progressão.

Pela Definição 2, analogamente ao processo efetuado para determinar a relação de recorrência relacionada a progressões aritméticas, obtemos (9):

$$
a_{n}=a_{1} \cdot q^{n-1}
$$

Tal qual em uma PA, conhecendo-se o primeiro termo e a razão da $P G$, podemos escrever qualquer termo da sequência. De forma análoga ao visto para a PA, podemos calcular um termo de uma PG a partir de qualquer outro, mediante a relação $a_{n}=a_{m} \cdot q^{n-m}$. 
2.6.1 Soma dos termos de uma PG finita

A soma $S_{n}$ dos $n$ primeiros termos de uma progressão geométrica finita de razão $q$ pode ser escrita conforme indicado em (10).

$$
S_{n}=a_{1}+a_{2}+\ldots+a_{n-1}+a_{n}
$$

Multiplicando ambos os membros de (10) pela razão $q$, obteremos (11):

$$
S_{n} \cdot q=a_{1} \cdot q+a_{2} \cdot q+\ldots+a_{n-1} \cdot q+a_{n} \cdot q
$$

Pela definição de PG, temos que $a_{n-1} \cdot q=a_{n}$. Desta forma, (11) pode ser reescrita conforme indicado em (12).

$$
S_{n} \cdot q=a_{2}+a_{3}+\ldots+a_{n-1}+a_{n}
$$

Subtraindo ordenadamente os termos de (10) dos termos de (12), obtemos (13).

$$
S_{n} \cdot q-S_{n}=a_{n} \cdot q-a_{1}
$$

Isolando $S_{n}$ obteremos (14), que fornece a expressão para o cálculo da soma dos $n$ primeiros termos de uma PG finita.

$$
S_{n}=\frac{a_{n} q-a_{1}}{q-1}
$$

Note-se que a soma dos $n$ primeiros termos de uma PG depende da razão, do primeiro e do último termo. No entanto, substituindo (9) em (14), podemos reescrevê-la conforme indicado em (15).

$$
S_{n}=\frac{a_{1}\left(q^{n}-1\right)}{q-1}
$$

Assim, a soma dos $n$ primeiros termos de uma PG também pode ser efetuada com base na razão e no primeiro termo.

\subsection{Regime de Capitalização Simples}

Iezzi; Hazzan e Degenszajn (2004, p. 44) definem o regime de capitalização simples como sendo o regime no qual os juros de cada período são constantes e dados pelo produto do capital emprestado pela taxa mensal de juros. Desta forma, se um capital $C$ é emprestado à taxa mensal $i$, os juros $J(n)$ devidos depois de $n$ meses após o empréstimo são dados por (16).

$$
J(n)=C . i . n
$$

Desta forma, o montante da dívida, ou seja, a soma dos juros com o capital emprestado é dada por (17).

$$
M(n)=C(1+i n)
$$

Utilizando a notação de sequências, podemos reescrever (16) e (17) como $J_{n}=C . i . n$ e $M_{n}=C(1+i . n)$, respectivamente. Com isso, fazendo a diferença entre dois termos consecutivos da sequência formada pelos juros e a diferença entre dois termos consecutivos da sequência formada pelos montantes, verificamos que as sequências numéricas formadas são duas PAs de razão igual a $C . i$.

É importante destacar que Morgado; Wagner e Zani (2001, p. 56-57) não dão ênfase ao conceito de juros simples no livro Progressões e Matemática Financeira. No entanto, destaca-se o fato de que os juros simples são tipicamente utilizados no cálculo dos juros de mora, ou seja, nos juros cobrados em pequenos atrasos de pagamento.

\subsection{Regime de Capitalização Composta}

No regime de capitalização composta, o montante num período qualquer $k$ é obtido aplicando a taxa periódica $i$ sobre o montante do período 
imediatamente anterior. Desta forma, podemos definir o regime de capitalização composta pelo teorema a seguir.

Teorema 1: No regime de capitalização composta, cuja taxa periódica é $i$, o valor inicial $C_{0}$ se transforma no montante $M_{n}=C_{0}(1+i)^{n}$ após decorridos $n$ períodos de tempo (MORGADO; WAGNER, ZANI, 2001, p. 45).

Demonstração: Seja $M_{k}$ o montante de uma aplicação após decorridos $k$ períodos de tempo. Após decorridos $k+1$ períodos de tempo, o montante $M_{k+1}$ é tal que $M_{k+1}=M_{k}+i \cdot M_{k}$. Desta forma, teremos que $M_{k+1}=M_{k}(1+i)$.

A recorrência com a qual é possível calcular os montantes para qualquer instante de tempo $k$ é uma progressão geométrica de razão $q=1+i$. Assim, o montante após decorridos $n$ períodos será dado por (18).

$$
M_{n}=C_{0}(1+i)^{n}
$$

Desta forma, percebe-se que a sequência numérica formada pelos montantes em cada período será uma progressão geométrica de razão $1+i$, podendo ainda ser escrita na forma de função conforme indicado em (19).

$$
M(n)=C_{0}(1+i)^{n}
$$

\section{$2.9 \mathrm{O}$ valor do dinheiro ao longo do tempo}

Segundo Morgado; Wagner e Zani (2001, p. 44-45), são exemplos de erros comuns em raciocínios financeiros:

a) Achar que quantias monetárias maiores valem mais que menores; b) Achar que uma determinada quantia possui sempre o mesmo valor;

c) Somar quantias referidas a épocas diferentes.

A seguir, apresentamos exemplos para cada um dos erros de raciocínio anteriormente elencados.

EXEMPLO 1: O valor de $\mathrm{R} \$ 110,00$ pode valer mais, menos ou o mesmo que $\mathrm{R} \$$ 100,00. Podemos tomar como exemplo a aplicação de $\mathrm{R} \$ 100,00$ na caderneta de poupança, na qual renderia juros e após um determinado período poderia valer mais que $\mathrm{R} \$ 110,00$.

EXEMPLO 2: $\mathrm{R} \$ 100,00$ pode ter valor significativo superior ou inferior a essa quantia. Podemos tomar como exemplo um cenário econômico com inflação, onde o valor de $\mathrm{R} \$ 100,00$ na data de hoje terá poder aquisitivo menor após determinado período de tempo.

EXEMPLO 3: A compra de um bem em 6 prestações iguais de $\mathrm{R} \$ 51,00$ pode ser mais vantajosa do que comprar o bem em 3 prestações de $R$ \$100,00. Isso porque o valor para pagamento à vista pode ser investido em uma aplicação financeira, rendendo juros.

Nas três situações, o fator primordial para entender onde está o erro é considerar o valor do dinheiro ao longo do tempo, ou seja, sua equivalência em épocas (ou datas focais) diferentes. Morgado; Wagner e Zani (2001, p. 46) definem (20) como sendo a fórmula fundamental para a equivalência de capitais, onde $F$ é o valor futuro, $A$ é o valor atual, $i$ a taxa de juros e $n$ o período de tempo.

$$
F=A(1+i)^{n}
$$

Isso se deve ao fato de que para obtermos o valor atual de uma quantia 
antes de transcorridos $n$ períodos de tempo, basta dividir o valor futuro por $(1+i)^{n}$ (ou multiplicar por $\left.(1+i)^{-n}\right)$. O inverso é feito para se calcular o valor futuro: multiplica-se o valor atual por $(1+i)^{n}$.

A seguir, apresentamos um exemplo com a aplicação deste conceito. EXEMPLO 4: Retomando a situação apresentada no Exemplo 3, considerando o valor do dinheiro como $0,5 \%$ ao mês, e tomando o vencimento da primeira prestação como sendo 30 dias após a compra, vamos analisar qual das opções é a mais vantajosa.

SOLUÇÃO: Calculando a atualização do valor total da compra em 6 prestações de $\mathrm{R}$ \$ 51,00 considerando o momento em que o bem é adquirido como sendo a época 0 , obteremos a soma $\frac{51}{1,005^{1}}+\frac{51}{1,005^{2}}+\ldots+\frac{51}{1,005^{5}}+\frac{51}{1,005^{6}}, \quad$ ou seja, o valor atual da quantia será aproximadamente $\mathrm{R} \$ 292,26$.

Analogamente, o valor atual do montante para a compra em 3 prestações de $\mathrm{R} \$ 100,00$, considerando o momento da compra como sendo a época 0 , será dada por $\frac{100}{1,005^{1}}+\frac{100}{1,005^{2}}+\frac{100}{1,005^{3}}$, ou seja, aproximadamente $\mathrm{R} \$ 295,07$. Desta forma, seria mais vantajoso comprar o bem parcelado em 6 vezes.

Com relação à análise sobre o método mais vantajoso de se efetuar uma compra (à vista ou parcelado), os cálculos, embora simples, são um tanto trabalhosos para efetuação à mão, sendo necessário recorrer a uma calculadora científica. Além disso, sobre sua resolução podemos considerar os seguintes questionamentos: a) E se fosse necessário decidir de forma rápida sobre qual a melhor proposta, como fazer?

b) E se o parcelamento fosse em mais vezes, digamos 12 prestações?

c) E se houver desconto para pagamento à vista, ainda assim vale a pena comprar parcelado?

Podemos obter as respostas para esses questionamentos utilizando, mais uma vez, progressões geométricas. Conforme disposto no Exemplo 4, o cálculo do valor atual $(A)$ de um bem parcelado em $n$ parcelas de $P$ reais, com a primeira parcela vencendo 30 dias após a compra, com o dinheiro valendo $i$ ao mês corresponde à soma dos termos de uma PG de razão $(1+i)^{-1}$, de primeiro termo igual a $\frac{P}{1+i}$, cujo valor é dado por (21).

$$
A=\sum_{j=1}^{n} \frac{P}{(1+i)^{j}}=\frac{P}{1+i} \cdot \frac{\left[(1+i)^{-1}\right]^{n}-1}{(1+i)^{-1}-1}
$$

Simplificando a expressão, o valor de $A$ é dado por (22).

$$
A=P \frac{1-(1+i)^{-n}}{i}
$$

No caso de haver desconto para a compra à vista, essa análise também pode ser utilizada, conforme apresentado no exemplo a seguir.

EXEMPLO 5: Um aparelho de televisão custa $R$ \$ 500,00, valor que pode ser parcelado em até 4 prestações iguais e mensais, com a primeira vencendo 30 dias após a compra. No caso de compra à vista, é dado o desconto de $5 \%$ sobre o valor do bem. Considerando que o dinheiro vale $0,5 \%$ ao mês, qual a opção mais vantajosa: comprar à vista ou parcelado? 
SOLUÇÃO: Nesse caso, o desconto dado é de 5\% sobre $\mathrm{R} \$ 500,00$, sendo o preço para compra à vista igual a $\mathrm{R} \$$ 475,00 . Calculando a atualização do valor do aparelho de televisão considerando o momento da compra como sendo a época 0 , usando (22) teremos que $A=100 \frac{1-1,005^{-5}}{0,005}$, ou seja, aproximadamente R\$492,59. Desta forma, é mais vantajoso comprar à vista.

Dependendo da taxa do valor mensal do dinheiro, a análise pode ter resultado diferente. No Exemplo 5, caso o valor mensal do dinheiro fosse $2 \%$ ao mês, o valor atual na época 0 seria dado por $A=100 \frac{1-(1,02)^{-5}}{0,02}$, ou seja, $\mathrm{R} \$$ 471,35 . Neste caso, seria mais vantajoso comprar a prazo.

\subsection{Planilhas eletrônicas no ensino de matemática}

Entre as diversas possibilidades tecnológicas disponíveis, optamos por estruturar uma proposta de atividades por meio da utilização de planilhas eletrônicas, com o uso do software Microsoft Excel $^{\circledR}$. Tal escolha se justifica pelo fato de ser um programa de fácil acesso e manipulação, tanto pelos alunos quanto pelo professor.

Um dos possíveis usos dessa ferramenta é a construção de calculadoras, por meio da qual podem ser utilizadas situações-problema como base para sua elaboração. Cabe ressaltar que a funcionalidade dos simuladores propostos é similar à funcionalidade da calculadora convencional, utilizada para efetuar cálculos com números de diversos algarismos significativos. $\mathrm{O}$ computador exercerá o papel de facilitador no sentido de poupar tempo na execução dos cálculos, além de possibilitar a exploração de propriedades e características dos objetos de estudo.

Desta forma, situações-problema poderão ser traduzidas em modelos matemáticos, os quais podem ser transcritos para as planilhas eletrônicas. Essa abordagem favorece também a exploração de operações algébricas na manipulação das fórmulas obtidas na modelagem das soluções das situaçõesproblema.

Giraldo; Caetano e Mattos (2012, p.26), afirmam que dentre os recursos disponíveis nas planilhas eletrônicas se destacam:

a) A manipulação e operações com grandes quantidades de dados numéricos;

b) A articulação entre diferentes formas de representação;

c) As ferramentas lógicas;

d) As ferramentas estatísticas.

Dentre os recursos citados pelos autores, os três primeiros exercem papel fundamental como facilitadores no estudo de matemática financeira, pois em muitas situações sobre a temática os cálculos envolvem potências numéricas, incógnitas na forma de expoente e análise de situações envolvendo diferentes cálculos, o que pode acarretar no uso de um intervalo de tempo significativo para sua conclusão.

\section{Simuladores e planilhas eletrônicas}

Quando os alunos do Ensino Médio tem os contatos inicias com a simbologia algébrica, é comum a dificuldade com relação ao significado dos símbolos. Nesse aspecto, a experiência 
de codificação e manipulação da simbologia utilizada nas planilhas eletrônicas, em especial a verificação de erros de codificação, podem auxiliar os alunos a entenderem o significado e regras dos símbolos utilizados (GIRALDO; CAETANO E MATTOS, 2012, p.26).

Nesse contexto, em cada situação-problema tratada será relevante que o aluno esteja apto a resolver equações na forma algébrica, de forma que não seja o professor o responsável por sintetizar as respostas para cada simulador, mas o aluno estimulado para tal feito. Espera-se que com isso, o aluno seja instigado a explorar e conhecer outras relações entre as diferentes variáveis que compõe as soluções das situaçõesproblema analisadas.

A seguir, apresentamos sugestões de simuladores que podem ser elaborados pelos alunos após a exploração algébrica de algumas das fórmulas utilizadas na temática financeira.

\subsection{Simuladores envolvendo a relação $C_{n}=C_{0}(1+i)^{n}$}

Conforme vimos anteriormente, se um capital $C_{0}$ é aplicado à taxa mensal $i$ no regime de juros compostos, a função que exprime o montante após $n$ meses é dado por (19). Baseados nessa função, descreveremos a seguir a elaboração de dois simuladores.

\subsubsection{Simulador 1 - Cálculo do montante após $n$ meses}

Dados o capital inicial, a taxa mensal de remuneração e o número de depósitos a serem efetuados, o simulador calculará o montante gerado após ser efetuado o último depósito. Este si- mulador é baseado na utilização direta de (19).

\section{PROCEDIMENTOS NECESSÁRIOS:}

1) Na célula $A 1$, digita-se Capital inicial;

2) Na célula $A 2$, digita-se Taxa mensal;

3) Na célula $\mathrm{A} 3$, digita-se Número de meses;

4) Na célula A4, digita-se Montante;

5) $\mathrm{Na}$ célula $\mathrm{B} 4$, digita-se a fórmula $=\mathrm{B} 1^{*}(1+\mathrm{B} 2)^{\wedge} \mathrm{B} 3$.

As células B1, B2 e B3 servirão como campo de entrada para os valores das variáveis da coluna A. Ao serem preenchidas, o montante será apresentado na célula B4.

Por se tratar de campos de saída de dados de diferentes formatos, o layout de algumas das células da coluna $B$ pode ser adequado às características do resultado a ser visualizado. Neste exemplo, o texto das células B1 e B4 foi alterado para o formato de moeda.

Com essa formatação e modificando a cor do plano de fundo das células da coluna A para cinza, o simulador apresentará a disposição apresentada na Figura 1.

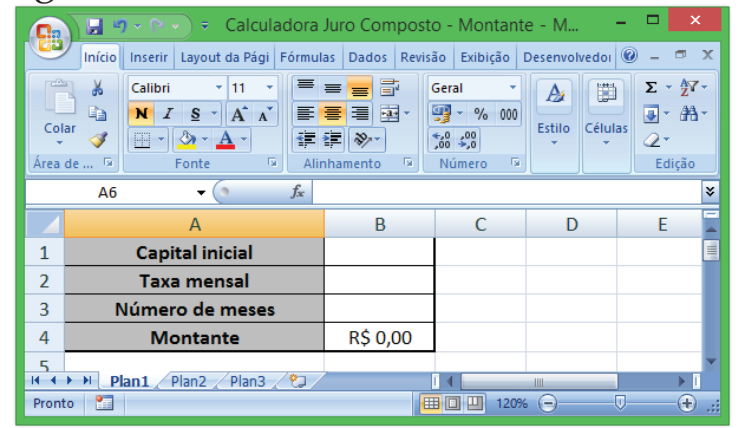

Figura 1: Layout do Simulador 1

A Figura 2 ilustra a utilização do Simulador 1 para o cálculo do montante gerado pelo capital de $\mathrm{R} \$ 150,00$, aplicado a $12 \%$ ao mês, ao final de 3 meses do momento da aplicação. 


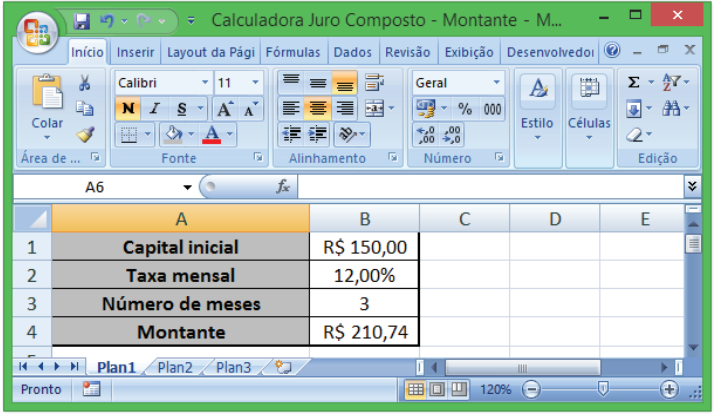

Figura 2: Exemplo de utilização do Simulador 1

\subsubsection{Simulador 2 - Tempo para evolu- ção do capital inicial}

Efetuando manipulações algébricas em (19), podemos calcular o tempo necessário para se atingir determinado montante. Seja $M$ o valor do montante para o qual o capital inicial $C_{0}$ deva evoluir, se aplicado à taxa mensal igual a $i$. Calculando o tempo $t$ necessário para que isso ocorra, obteremos a igualdade $C_{0}(1+i)^{t}=M$. Dividindo ambos os membros por $C_{0}$, obteremos que $(1+i)^{t}=\frac{M}{C_{0}}$.

Utilizando logaritmos em ambos os membros, e isolando $t$, temos o resultado apresentado em (23).

$$
t=\frac{\ln \frac{M}{C_{0}}}{\ln (1+i)}
$$

Utilizando (23), podemos elaborar o simulador para o cálculo do tempo.

\section{PROCEDIMENTOS NECESSÁRIOS:}

1) Na célula A1, digita-se Capital inicial;

2) Na célula $A 2$, digita-se Montante;

3) Na célula A3, digita-se Taxa mensal;

4) Na célula A4, digita-se Número de meses;

5) $\mathrm{Na}$ célula $\mathrm{B} 4$, digita-se a fórmula $=\ln (\mathrm{B} 2 / \mathrm{B} 1) / \ln (1+\mathrm{B} 3)$.

Assim como no Simulador 1, as células B1, B2 e B3 serão os campos de entrada para os valores das variáveis da coluna A. A célula B4 será o campo de saída, onde será registrado o número de meses. Formatando as células, chegamos à visualização indicada na Figura 3.

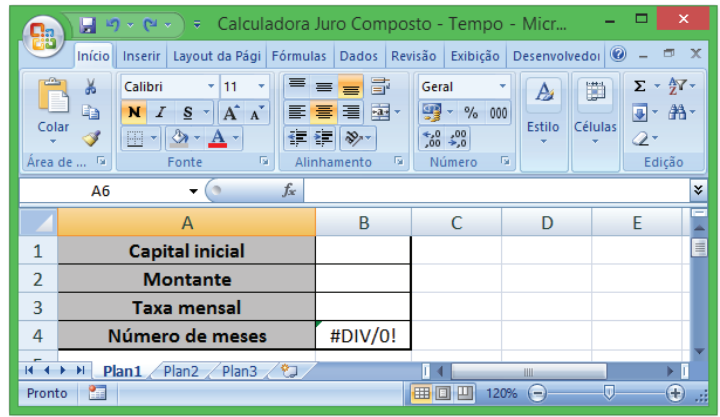

Figura 3: Layout do Simulador 2

Note-se que ao digitar as fórmulas sem inserir valores nas células B1 a B3, aparece a mensagem \#DIV/0! na célula B4. Esse fato pode ser aproveitado para questionar os alunos sobre a razão disto acontecer, que obviamente é o fato do software considerar o valor de cada célula como sendo igual a zero. Como a resolução é em termos de logaritmos, o erro acontece devido ao fato de ocorrer uma divisão por zero, uma vez que $\ln (1+\mathrm{B} 3)$ corresponderia a $\ln 1$, que é igual a zero.

Note-se, também, que com esse simulador podemos efetuar simulações de multiplicação do capital inicial aplicado a uma determinada taxa de forma empírica, bastando para isso inserir um valor qualquer como capital inicial e o valor que se deseja obter no campo de entrada do valor do montante a ser atingido.

Por exemplo, utilizando o capital inicial igual a $\mathrm{R} \$ 100,00$, o montante igual a $\mathrm{R} \$ 200,00$ e a taxa mensal de rendimento de $8 \%$, verificamos que o tem- 
po necessário para que o capital inicial dobre é superior a 9 meses, conforme indicado na Figura 4.

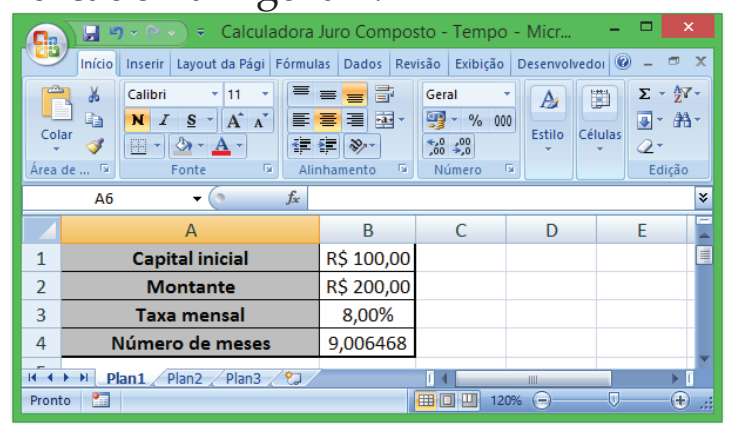

Figura 4: Exemplo de utilização do Simulador 2

Com a exploração de outros exemplos numéricos envolvendo o montante igual ao dobro do capital inicial será possível verificar que o tempo necessário para que o capital inicial duplique não será alterado.

\subsection{Simuladores de financiamento}

Embora não seja complicada a elaboração de um simulador que permita calcular o valor de todas as parcelas de um financiamento, optamos por sintetizar as informações das modalidades SAC e Price em dois pequenos simuladores, conforme descrições adiante.

3.2.1 Simulador 3 - Financiamento pelo sistema de amortização constante (SAC)

O Sistema de Amortização Constante (SAC), como a própria denominação aponta, possui como característica principal a amortização constante da dívida referente ao valor financiado, sendo os juros de cada período calculados sobre o saldo devedor do mês imediatamente anterior. A amortização mensal é calculada pelo quociente entre o valor numérico da quantia financiada e do período de tempo do financiamen- to, que equivale ao número de parcelas a serem pagas.

Sendo $F$ o valor financiado, e $n$ o número de parcelas, o valor da amortização mensal $A$ será igual a $\frac{F}{n}$. Indicando por $P_{k}$ o valor da parcela a ser paga no $k$-ésimo mês, $J_{k}$ os juros a serem pagos junto em $P_{k}$, e $i$ a taxa mensal de juros do financiamento, teremos que $P_{k}=A+J_{k}$.

As séries que descrevem o valor dos juros e o saldo devedor são progressões aritméticas. A sequência do saldo devedor é uma PA de razão $r_{1}=-\frac{F}{n}$. Devido a isso, a sequência dos juros mensais também é uma PA, porém, de razão $r_{2}=-\frac{F i}{n}$. Temos também que o saldo devedor no $k$-ésimo mês será igual a $\frac{F(n-k)}{n}$.

Desta forma, podemos facilmente calcular o total de juros pagos no financiamento. A forma mais simples para efetuar esse procedimento consiste em efetuar a soma dos termos da PA formada pelos juros mensais. Sendo $J$ o total de juros pagos durante o financiamento, utilizando (8), obteremos (24):

$$
J=\frac{F i n+F i}{2}
$$

A seguir, vemos como utilizar essa fórmula para calcular os juros em um financiamento pelo SAC.

EXEMPLO 6: Calcular o valor dos juros a serem pagos no financiamento de $\mathrm{R} \$$ 1000,00 em 5 parcelas pelo SAC, à taxa de $1 \%$ ao mês. 
SOLUÇÃO: Os juros a serem pagos são dados por $J=\frac{1000.0,01.5+1000.0,01}{2}$, ou seja, aproximadamente $\mathrm{R} \$ 30,00$.

O entendimento sobre tal modalidade de financiamento é relevante no sentido de que esta é a comumente utilizada para o financiamento de imóveis. Desta forma, é possível desenvolver atividades com situações que eventualmente possam ocorrer no cotidiano dos alunos ou de suas famílias.

Para elaboração do simulador do SAC, utilizaremos as seguintes informações:

I - O juro mensal a ser pago é calculado sobre o saldo devedor no momento do pagamento da parcela;

II - A amortização mensal da dívida (A) é igual à divisão do valor financiado pelo número de parcelas do financiamento;

III - Os valores das parcelas do financiamento são decrescentes, na forma de uma progressão aritmética de razão $-A i$.

Pela informação I, expressamos a parcela inicial por $C\left(1+\frac{i}{12}\right)$, onde $C$ é o valor financiado e $i$ é a taxa anual de juros cobrada. Da mesma forma, a parcela final será igual a $A\left(1+\frac{i}{12}\right)$, pois no momento da última parcela a dívida é igual à amortização mensal.

Pela informação II, expressamos o valor da amortização mensal da dívida como sendo $\frac{C}{n}$, onde $n$ é o número de parcelas do financiamento.

Pela informação III, conseguimos expressar o total de juros do finan- ciamento como a soma de uma PA com $n$ termos, na qual o primeiro termo é igual a $C\left(1+\frac{i}{12}\right)$ e o último é igual a $A\left(1+\frac{i}{12}\right)$, utilizando (8).

Os passos necessários para a elaboração do simulador são descritos a seguir.

PROCEDIMENTOS NECESSÁRIOS:

1) Na célula A1, digitamos Valor financiado;

2) Na célula $\mathrm{A} 2$, digitamos Número de parcelas;

3) Na célula A3 digitamos Taxa anual de juros anual;

4) Na célula A4 digitamos Amortização mensal;

5) Na célula A5 digitamos Parcela inicial;

6) Na célula A6 digitamos Parcela final;

7) Na célula A7 digitamos Total de juros;

8) Na célula A8 digitamos Total do financiamento;

$\mathrm{Na}$ coluna $\mathrm{B}$, teremos as células B1, B2 e B3 como campos de entrada. Nas demais digitamos as fórmulas:

9) $\mathrm{Na}$ célula $\mathrm{B} 4$, digitamos a fórmula =B1/B2;

10) Na célula $B 5$, digitamos a fórmula $=\mathrm{B} 1^{*}(1+\mathrm{B} 3 / 12)$;

11) Na célula $B 6$, digitamos a fórmula $=\mathrm{B} 4+\mathrm{B} 1 *(1+\mathrm{B} 3 / 12)$;

12) Na célula $B 7$, digitamos a fórmula $=(\mathrm{B} 5+\mathrm{B} 6)^{*} \mathrm{~B} 2 / 2-\mathrm{B} 1$;

13) Na célula $B 8$, digitamos a fórmula $=\mathrm{B} 1+\mathrm{B} 7$.

Formatando as células adequadamente, teremos o layout apresentado na Figura 5. 


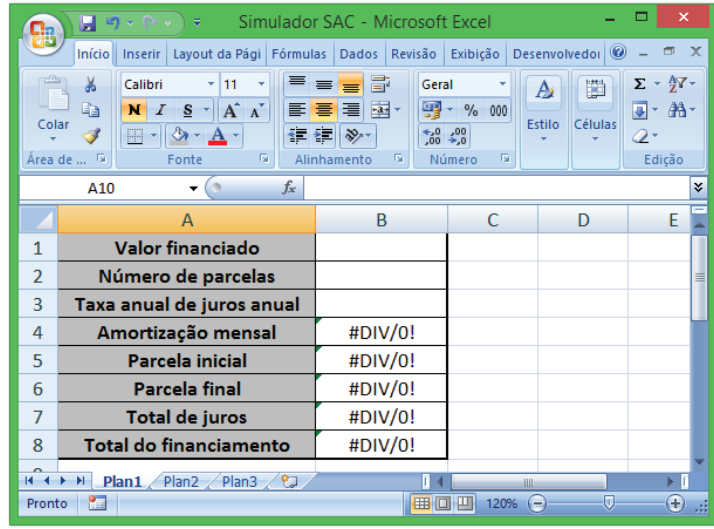

Figura 5: Layout do Simulador 3

A Figura 6 ilustra a utilização do Simulador 3 para o cálculo das parcelas final e inicial, amortização mensal e o total de juros referente ao financiamento de $\mathrm{R} \$$ 100.000,00 no SAC em 10 anos, com taxa de $12 \%$ ao ano.

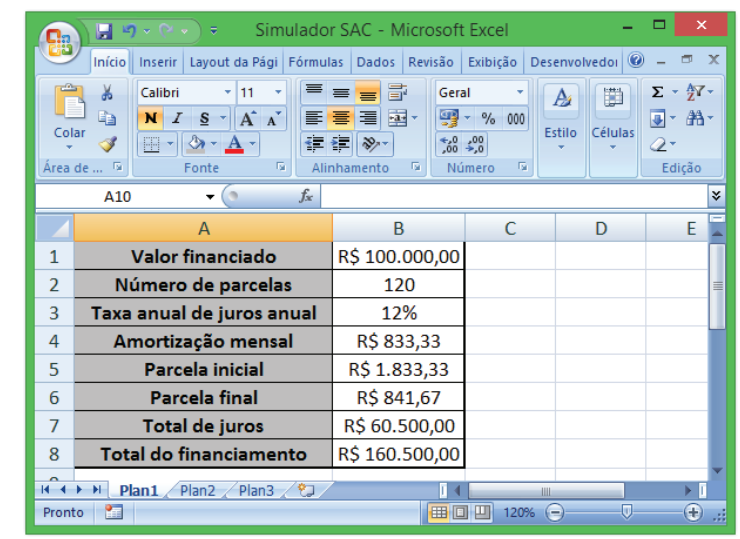

Figura 6: Exemplo de utilização do Simulador 3

Com a recente expansão e facilidade na obtenção de crédito habitacional, muitas famílias têm a oportunidade de adquirir ou construir um imóvel, deixando de pagar aluguel e investindo o dinheiro num imóvel próprio. Essa temática permite a exploração de diversas situações-problema.

No ilustrado pela Figura 6, é importante notar que na parcela inicial são pagos exatamente $\mathrm{R} \$ 1000,00$ de juros, valor significativamente superior à amortização mensal. Aproveitando essa observação, podem ser exploradas situações onde é necessário decidir se é mais vantajoso financiar um imóvel ou se é mais atrativo continuar a pagar aluguel e comprar um imóvel à vista.

Para isso, torna-se necessário o uso de mais de um tipo de simulador. Sem isso, a análise feita com a execução manual dos cálculos pode se tornar pouco atrativa para os alunos.

3.2.2 Simulador 4 - Financiamento pelo método Price

No sistema de amortização francês, ou sistema Price, as parcelas referentes ao financiamento de uma determinada quantia são constantes e iguais a um valor $P$. A ideia utilizada para se determinar os valores das parcelas está atrelada à ideia de equivalência de capitais.

Seja $F$ o valor financiado, $P$ o valor da parcela mensal do financiamento e $i$ a taxa mensal de juros. Como todas as parcelas serão iguais, o valor atual da quantia financiada é igual à equivalência das parcelas na data focal zero, considerando o pagamento da primeira parcela um mês após a contratação do financiamento, conforme indicado em (25).

$$
\begin{aligned}
& \quad F=\frac{P}{1+i}+\frac{P}{(1+i)^{2}}+\ldots++\frac{P}{(1+i)^{n}} \\
& F \text { é equivalente à soma dos } \mathrm{n} \\
& \text { primeiros termos de uma progressão } \\
& \text { geométrica de razão } \frac{1}{1+i} \text { e } a_{1}=\frac{P}{1+i} .
\end{aligned}
$$
Desta forma, utilizando (22), $F$ pode ser calculado por (26).

$$
F=P \frac{1-(1+i)^{-n}}{i}
$$

Assim, o valor de $P$ é dado por (27).

$$
P=F \frac{i}{1-(1+i)^{-n}}
$$


Utilizando (27) podemos elaborar um simulador para o sistema de financiamento pelo método Price, no qual são calculados a parcela mensal e o total de juros a serem pagos durante o financiamento.

\section{PROCEDIMENTOS:}

1) Na célula A1, digitamos Valor financiado;

2) Na célula A2, digitamos Taxa de juros mensal;

3) Na célula $A 3$, digitamos Número de parcelas;

4) $\mathrm{Na}$ célula A4, digitamos Valor da parcela;

5) Na célula A5, digitamos Total de juros;

6) Na célula A6, digitamos Total do financiamento;

7) Na célula $\mathrm{B} 4$, digitamos a fórmula $=\mathrm{B} 1{ }^{*} \mathrm{~B} 2 /\left(1-(1+\mathrm{B} 2)^{\wedge}-\mathrm{B} 3\right)$;

8) Na célula $\mathrm{B} 5$, digitamos a fórmula $=\mathrm{B} 3{ }^{*} \mathrm{~B} 4-\mathrm{B} 1$;

9) $\mathrm{Na}$ célula $\mathrm{B} 6$, digitamos a fórmula $=\mathrm{B} 3{ }^{*} \mathrm{~B} 4$.

As células B1 a B3 serão campos de entrada dos valores das variáveis relacionadas na coluna $\mathrm{A}$, e as células $\mathrm{B} 4$ a B6 os campos de saída. Formatando as células utilizadas, obtemos o layout apresentado na Figura 7.

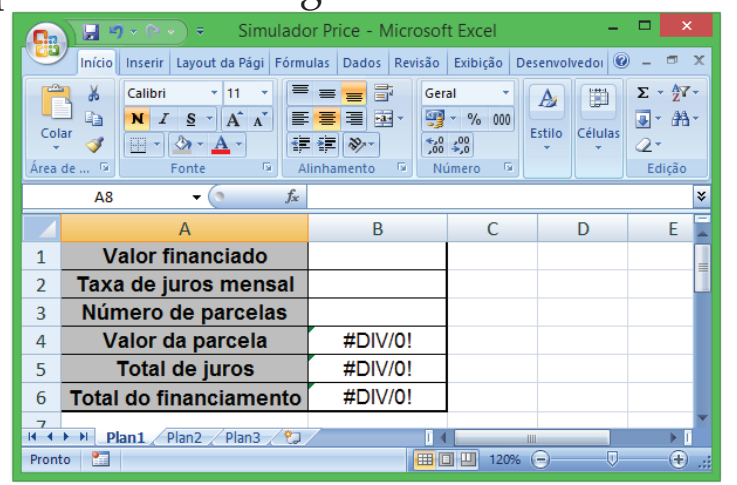

Figura 7 - Layout do Simulador 4

Neste simulador, o valor dos juros pagos no decorrer do financiamento foi calculado pela diferença entre o valor total do financiamento e o valor financiado.

$\mathrm{Na}$ figura 8, apresentamos um exemplo de utilização do Simulador 4 para o cálculo da parcela, juros e total do financiamento de $\mathrm{R} \$ 1000,00$ em 5 parcelas, à taxa de juros de $1 \%$ ao mês.

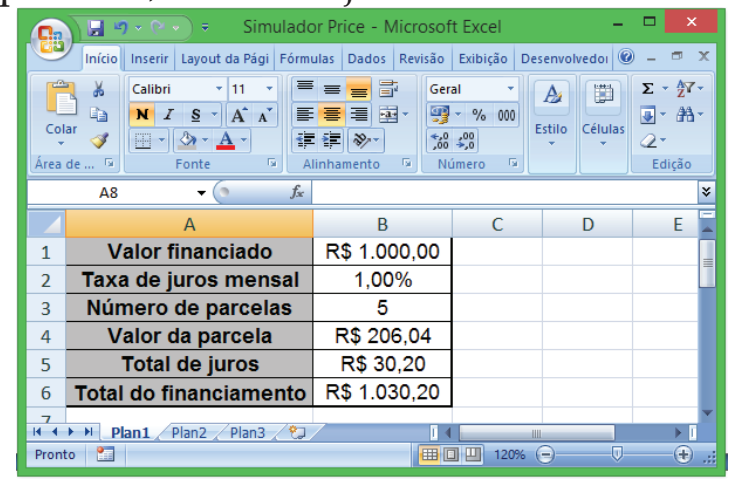

Figura 8: Exemplo de utilização do Simulador 4

\subsection{Comparativo entre os sistemas SAC e Price}

Em relação ao financiamento de uma determinada quantia, facilmente se percebe que os juros pagos no método SAC são inferiores aos juros pagos no financiamento pelo método Price.

No entanto, isso não é devido ao fato de uma modalidade de financiamento ser mais vantajosa em relação à outra, mas sim devido à amortização da dívida que é efetuada mensalmente.

Essas características são facilmente verificadas por meio da simulação simultânea do financiamento de um mesmo valor utilizando os simuladores 3 e 4 , utilizando a mesma taxa mensal de juros e o mesmo número de parcelas.

Este aspecto pode ser o motivador para a discussão sobre a justificativa do fato de o SAC ser normalmente utilizado para o financiamento de imóveis em detrimento ao uso do método Price, sendo oportuno frisar sobre as impor- 
tantes aplicações do último método em financiamentos de curto prazo, como parcelamento de veículos, eletrodomésticos, móveis e em empréstimos pessoais e consignados.

\section{Conclusões}

O presente trabalho teve como objetivo buscar respostas às formas de como o estudo da matemática financeira pode ser explorado na educação básica, em especial no Ensino Médio, com abordagens que relacionem o estudo de sequências, estimulem a educação financeira, e sejam idealizadas com a utilização de recursos tecnológicos.

Em consonância com a LDB e com o ponto de vista dos autores elencados no referencial teórico, vemos que a educação básica deve contemplar o pleno desenvolvimento do aluno para o mundo do trabalho e para o exercício da cidadania, razão pela qual as abordagens dos conteúdos devem ser pautadas no estudo das aplicações da teoria em situações reais significativas.

O bom entendimento sobre as práticas comerciais é de extrema relevância no estudo dessa temática, sendo recomendada a abordagem voltada para o entendimento das operações de empréstimo, podendo ser complementada com o entendimento sobre aplicações de capital, sendo imprescindível o estudo e reconhecimento dos padrões presentes nas séries numéricas relacionadas a esses temas.

As planilhas eletrônicas se apresentam como uma ferramenta útil nas aulas de matemática, e sua inserção no ambiente escolar deve ser planejada de forma cautelosa, de forma que não se sobreponha aos objetivos principais da disciplina.

Nesse contexto, a preparação técnico-didática do docente envolvido é fator preponderante, pois existe a possibilidade do software ser subutilizado, gerando um efeito inverso do esperado na aprendizagem e no envolvimento discente.

Se bem exploradas, as planilhas eletrônicas são eficientes no desenvolvimento de simuladores e calculadoras para estudo e análise de diversas situações reais, nas quais será possível aliar a exploração algébrica de fórmulas e a verificação de padrões na heurística das resoluções.

Desta forma, remetemo-nos ao princípio de que a educação escolar deve ser agente transformador do aluno, contextualizando o objeto de estudo com aplicações em situações reais significativas, visando contribuir para o exercício da cidadania, no sentido de que os indivíduos envolvidos possam gerir seus recursos de forma otimizada.

Com esse envolvimento, no qual é dada ênfase à participação discente, espera-se que a aprendizagem de tópicos de matemática financeira ocorra de maneira profícua e de forma significativa.

\section{Referências}

BRASIL. (1996) Ministério da Educação e Cultura. Lei no 9.394, de 20 de dezembro de 1996. Estabelece as diretrizes e bases da educação nacional. Brasília: MEC. Disponível em: $\langle$ http://www.planalto.gov.br/ccivil 03/lei s/19394.htm>. Acesso em: 20 jul. 2013.

. (2000) Ministério da Educação e Cultura. Orientações Educacionais Complementares aos Parâmetros Curriculares Nacionais (Ensino Médio): Ciências da Natureza, Matemá- 
tica e suas Tecnologias. Disponível em: $<$ http://portal.mec.gov.br/seb $>$. Acesso em: 10 ago. 2013.

. (2010) Ministério da República. Decreto no 7.397, de 22 de dezembro de 2010. Institui a Estratégia Nacional de Educação Financeira - ENEF, dispõe sobre a sua gestão e dá outras providências. Brasília. Disponível em: $<$ http://www.planalto.gov.br/ccivil 03/ Ato2 007-2010/2010/Decreto/D7397.htm>. Acesso em: 20 jul. 2013.

BORBA, M de C.; PENTEAdO, M.G. (2005). Informática e Educação Matemática. 3. ed. 1.reimp. Belo Horizonte: Autêntica, 100 p. (Coleção Tendências em Educação Matemática, 2)

DARWIN, I. F. (2012). Android Cookbook: Problemas e soluções para desenvolvedores Android. California: O'Reilly. 672 p.

DEITEL, P. J. (2013) Android: How To Program. New Jersey: Pearson Education, 873 p.

GIRALDO, V; CAETANO, P; MATTOS, F. (2012) Recursos Computacionais no Ensino de Matemática. Rio de Janeiro: SBM. 278 p. (Coleção PROFMAT, 06).

GRAVINA, M. A.; BASSO, M. V. de A. (2012) Mídias digitais na Educação Matemática. In: GRAVINA, et al. (Orgs.) Matemática, Mídias Digitais e Didática: tripé para formação do professor de Matemática. Porto Alegre: Evangraf. $180 \mathrm{p}$.

IEZZI, G; HAZZAN, S; DEGENSZAJN, D.M. (2004) Fundamentos de matemática elementar 11: matemática comercial, matemática financeira, estatística descritiva. 10. reimp. São Paulo: Atual, 232 p.

LIMA, E. L. (2007) Matemática e ensino. 3. ed. Rio de Janeiro: SBM. 207 p. (Coleção do Professor de Matemática, 16).

et al. (2006) A Matemática do Ensino Médio. v. 2. 6. ed. Rio de Janeiro: SBM. 308 p. (Coleção do Professor de Matemática, 14).

MORGADO, A. C.; WAGNER, E.; ZANI, S. (2001) Progressões e matemática financeira. 5. ed. Rio de Janeiro: SBM.121 p. (Coleção do Professor de Matemática, 8).
PARANÁ. (2008) Secretaria de Estado de Educação. Departamento de Educação Básica. Diretrizes Curriculares da Educação Básica Matemática. Curitiba: SEED. Disponível em: $<\mathrm{http}$ ://www.educadores.diaadia.pr.gov.br/a rquivos/File/diretrizes>. Acesso em: 20 jul. 2013.

PENTEADO, M. G. (2000) Possibilidades para a formação de professores de matemática. In: ; BORBA, M. de C. (Orgs.). A informática em ação: formação de professores, pesquisa e extensão. São Paulo: Olho d'Água. $79 \mathrm{p}$.

SANTOS, R. P. dos. (2011) Uma proposta de formação continuada sobre matemática financeira para professores de matemática do Ensino Médio. Dissertação (Mestrado Profissional em Educação Matemática) - Universidade Severino Sombra, Vassouras.

SOUSA, L de. (2012) Resolução de problemas e simulações: investigando potencialidades e limites de uma proposta de educação financeira para alunos do Ensino Médio de uma escola da rede privada de Belo Horizonte (MG). Dissertação (Mestrado Profissional em Educação Matemática) - Universidade Federal de Ouro Preto, Ouro Preto. 